\title{
Ciertas condiciones son tratadas menos frecuentemente en pacientes con enfermedades crónicas
}

The treatment of unrelated disorders in patients with chronic medical diseases. Redelmeier DA, Tan SH, Booth GL. N Engl J Med 1998;338:1516-20

\section{Objetivo}

Evaluar la hipótesis de que los pacientes con ciertas enfermedades tienen menos probabilidades de recibir tratamiento si, concurrentemente, tienen enfermedades médicas crónicas.

\section{Diseño}

Estudio de corte transversal.

\section{Lugar}

Ontario, Canadá.

\section{Pacientes}

Todos los residentes de Ontario, mayores de 65 años que recibían tratamiento para las enfermedades crónicas estudiadas: diabetes mellitus (DM), enfermedad pulmonar obstructiva crónica (EPOC) y síndromes psicóticos identificados a través de la prescripción de insulina, bromuro de ipratropio y haloperidol respectivamente. Para cada una de estas enfermedades crónicas se seleccionó un tratamiento no relacionado directa ni indirectamente con la misma. El tratamiento era bien definido y menos importante pero no trivial. Los tratamientos seleccionados fueron terapia de reemplazo hormonal (TRH) para los pacientes con DM, hipolipemiantes para los pacientes con EPOC y tratamiento para la artritis para los pacientes psicóticos.

El estudio incluyó 1.344 .145 pacientes ( $56 \%$ mujeres, edad media de 74 años). De ellos, 1.226 .064 (91\%) recibían al menos una medicación ( $58 \%$ de éstos eran mujeres, edad media de 74 años).

\section{Resultados}

Los pacientes con DM recibían menos terapia de reemplazo hormonal que otros pacientes ( $2.4 \%$ vs. $5.9 \%, P<0.001)$. En otras palabras, la DM se asoció a un $60 \%$ menos de "riesgo" de recibir TRH (OR* 0.40 , IC95\% 0.37-0.43).

Los pacientes con EPOC recibían menos medicación hipo lipemiante que los otros pacientes ( $6.3 \%$ vs. $8.7 \%, P<0.001)$. Es decir, el EPOC estuvo asociado con un $31 \%$ menos de 'riesgo' de recibir hipolipemiantes $\left(0 R^{*} 0.69\right.$, IC95\% 0.67-0.72).

Similarmente los pacientes psicóticos recibían menos tratamiento de la artritis que los otros pacientes ( $18 \%$ vs. $27 \% \mathrm{P}<0.001,0 R^{*} 0.59$, IC $950.57-0.62$ ).

Tabla 1. Sumario del Análisis

\begin{tabular}{|c|c|}
\hline $\begin{array}{l}\text { Enfermedad Crónica y Tratamiento No relacionado } \\
\text { Reducción Relativa* }\end{array}$ & $\begin{array}{l}\text { Porcentaje de } \\
\text { (IC } 95 \%) \#\end{array}$ \\
\hline \multicolumn{2}{|l|}{ DM y TRH } \\
\hline Análisis básico & $60(57-63)$ \\
\hline Análisis multivariado† & $60(57-63)$ \\
\hline \multicolumn{2}{|l|}{ Análisis estratificado } \\
\hline Preparaciones orales & $60(57-63)$ \\
\hline Preparaciones transdérmicas & $55(40-66)$ \\
\hline Análisis de 1990 & $61(55-66)$ \\
\hline \multicolumn{2}{|l|}{ EPOC e hipolipemiantes } \\
\hline Análisis básico & $31(28-33)$ \\
\hline Análisis multivariado† & $23(20-26)$ \\
\hline \multicolumn{2}{|l|}{ Análisis estratificado T } \\
\hline Estatinas & $32(30-35)$ \\
\hline \multicolumn{2}{|l|}{ Agentes no estatínicos } \\
\hline \multicolumn{2}{|l|}{$21(16-26)$} \\
\hline Análisis de 1990 & $35(29-40)$ \\
\hline \multicolumn{2}{|l|}{ Síndromes psicóticos y tratamiento antiartrítico } \\
\hline Análisis básico & $41(38-43)$ \\
\hline Análisis multivariado $†$ & $38(35-40)$ \\
\hline \multicolumn{2}{|l|}{ Análisis estratificadoप } \\
\hline AINES & $41(39-43)$ \\
\hline DMA & $55(40-66)$ \\
\hline Análisis con datos de 1990 & $40(37-42)$ \\
\hline
\end{tabular}

\# Las reducciones relativas en el tratamiento están basadas en cálculos de odds-ratio*.

P 0.001 para todas las reducciones.

† Análisis ajustado para edad y sexo

पा En este caso se analiza según familia de drogas

AINEs = antiinflamatorios no esteroides. DMA $=$ drogas modificadoras de la artritis.

\section{Conclusiones}

En los pacientes de 65 años o más que tenían enfermedades médicas crónicas y que recibían medicación sin cargo, las enfermedades no relacionadas eran subtratadas.

\section{COMENTARIO}

La hipótesis de que los pacientes con una enfermedad crónica lo suficientemente importante como la diabetes, el EPOC o la psicosis suelen ser subtratados por otras condiciones es la conclusión final de este estudio que, por el tamaño de la muestra, difícilmente pueda ser refutada. No obstante, ciertas limitaciones merecen tenerse en cuenta: La probabilidad de falsos negativos, es decir diabéticos que no reciben. insulina, EPOC que no reciben ipratropio o psicóticos que no reciben haloperidol así como de falsos positivos, es decir individuos que reciben insulina, ipratropio o haloperidol y no son diabéticos, EPOC o psicóticos respectivamente, no está controlada. Asimismo el sobretratamiento de pacientes que no tienen estas enfermedades crónicas podría reducir la validez del estudio pero difícilmente explique totalmente las diferencias. El estudio no cuenta con información clínica de los pacientes ya que se hizo revisando la base de datos farmacológica de la provincia, que, como parte de un programa estatal, cubre la medicación prescripta en forma gratuita. Por lo tanto, los motivos por los cuales esta población aparenta estar menos tratada por condiciones concomitantes (dislipidemia, menopausia, artritis) no pueden inferirse concluyentemente. El no tratamiento puede deberse a subdiagnóstico, decisión médica o de los pacientes. También el hecho de que esta población sea mayor de 65 años podría permitirnos suponer que los médicos y los pacientes, por razones varias como adherencia, interacciones o efectos adversos son más reticentes a polimedicar y en consecuencia eligen tratar el peor de los males. En este caso tres enfermedades serias fueron confrontadas con tres enfermedades o condiciones que podrían considerarse menos graves.

Deberemos también ser muy cautelosos a la hora de sacar conclusiones o trasladar automáticamente los resultados a nuestros pacientes. El precio de la medicación, la situación socioeconómica de la población o su situación urbana o rural, la fragmentación de los cuidados, son todos factores que pueden modificar sustancialmente las decisiones de tratamiento. Finalmente, este estudio confirma una impresión basada en la experiencia clínica: al enfrentarnos con pacientes con patologías graves que demandan un cuidado intenso uno puede pasar por alto otros problemas menos acuciantes pero aun así molestos para el paciente. De todas maneras, otra pregunta que no es respondida ni puede inferirse de este estudio es: ¿Son mejores los resultados si se tratan todas las enfermedades de un paciente confrontado al de tratar sólo la o las más graves? 